\title{
Reactive Navigation of a Mobile Robot Using a Hierarchical Set of Learning Agents
}

\author{
Frédéric Davesne \\ CEMIF-CSG-University of Evry \\ 40, r. Pelvoux, 91020 Evry Cdx, France. \\ davesne@cemif.univ-evry.fr
}

\begin{abstract}
Within the context of learning sequences of basic tasks to build a complex behavior, a method is proposed which uses a hierarchical set of incrementally learning agents. Each one has to respect a particular perceptive constraint. To do so, an agent must choose either to execute basic tasks or to call another agent in order to use its decision-making competences, according to its perception. The learning procedure of each agent is achieved by a reinforcement learning inspired algorithm based on an heuristic which does not need internal parameters. A validation of the method is given, using a simulated Khepera robot. A hierarchical set of 4 agents is created. Each one is dedicated to the exploitation of particular perceptive data. They use 5 basic tasks in order to achieve a goal-reaching behavior which is formulated by a high level strategy composed of logical rules using perceptive primitives.
\end{abstract}

\section{Introduction.}

\subsection{Development context.}

Within the framework of mobile robotics, it is often difficult to establish a relationship between the data perceived by the robot and the behavior it must achieve according to its input data.

Indeed, the perceptive data may be very noisy or may not be interpreted easily, so that modelling the mapping between perception and behavior could be a very difficult task. Reinforcement learning methods [1] have been widely used in that context [2],[3], mainly because they do not need a prior knowledge about the process model. Moreover, they theoretically achieve incremental learning and they can cope with a possible inertia of the system. But finding suitable internal

\author{
Claude Barret
}

\author{
CEMIF-CSG-University of Evry \\ 40, r.Pelvoux, 91020 Evry Cdx, France. \\ cbarret@cemif.univ-evry.fr
}

parameters for those algorithms is not intuitive and may be a difficult task [4]. Besides, it is not easy to find a compromise between the stability and the robustness of the algorithm and its incremental characteristic. Finally, given that the reinforcement methods need to sufficiently explore the perception space before finding a quite good solution, learning to fit a complex behavior in a reasonable lapse of time turns to be impossible without finding out some characteristics of the process, leading to a problem with a significantly decreased perception space. A solution could be to divide the whole task into coordinated sub-tasks [5], each one being easier to learn than the complex behavior. However, the problem is turned into another one: choosing to execute a precise sub-task is often tricky, especially if the choice depends on the perceptual data of the agent. In that case, applying a simple switching is not generally sufficient; the agent has to learn to decide which sub-task is to be executed according to its input data. Moreover, when a failure in the learning process occurs, one has to know if the cause of the mistake is due to a misleading choice of a sub-task or to an internal deficiency of the elected sub-task unit. In the last eventuality, it could be necessary to modify this unit to make it avoiding the same mistake. So, it must have the capacity to learn at anytime it is used: this is an important focus of incremental learning methods.

\subsection{Characteristics of the algorithm.}

The proposed algorithm has some hard links with the reinforcement learning concept: it is a trial/failure method, it does not need a prior knowledge of the process model, it copes with the temporal credit assignment problem and it is incremental. However, it is not based on an optimization method, but on the respect of binary perceptive constraints.

The framework of the algorithm is the collective learning of a hierarchical set of agents which aim is 


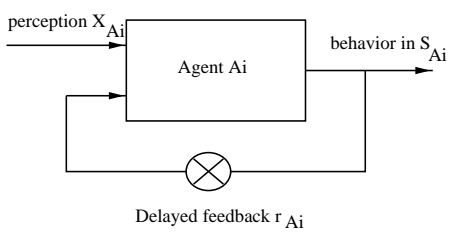

Figure 1: Diagram showing the links between one of the agents $A_{i}$ and its environment.

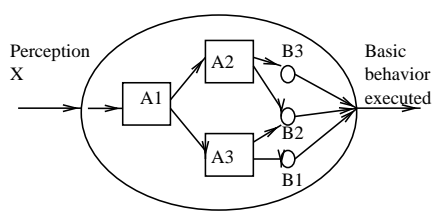

Figure 2: Hierarchical organization of the agents.

to respect their proper constraint given by a binary feedback signal (fig. 1). In order to achieve their goal, each agent may call another agent which is lower in the hierarchy or may decide to execute one of the basic behaviors fixed by the user before the learning process begins.

At each time, the chained decisional process is initiated by a master agent, ending with the execution of a basic behavior (fig. 2).

Each agent has to learn to decide according to its current perception. To do so, an internal representation of the influence of its choices (leading to the execution of a basic task) on its perception is built on-line by the meaning of an oriented graph which nodes are associated with a particular choice and a precise perceptive area. Each node has an internal binary value related to its quality according to the respect of the constraints of the agent. This value is obtained by a backpropagation of the values linked to the ending nodes of the graph (nodes associated with a failure) using a consistency law derivated from the minimax algorithm [6]. Here, an analogy is made with a two player game in which the agent must use some actions so that the response of the dynamic system (its opponent) never leads it to a losing state, that is to say a perceptive state in which the agent does not respect its constraints.

When a failure is given by the feedback signal of an agent whereas the other lower level agents have fulfilled theirs constraints, the first one attributes the mistake to its decision.

\subsection{Validation of the algorithm.}

The algorithm will be applied to a general goalseeking problem in which the obstacle avoidance is performed by a wall-following behavior. To do so, the mobile robot Khepera [7] simulator written par O.Michel [8], running on Unix-like operating systems, will be utilized, which allows to test the robustness of the algorithm in a very noisy perceptive data context. The incremental capability and the learning rapidity of the algorithm will be shown.

\section{Description of the algorithm.}

\subsection{Context and notations.}

The set of learning agents $S_{A}=\left\{A_{1}, A_{2}, \ldots, A_{m}\right\}$ receives a data stream which can be modelled by a vector $X=\left(X^{1}, X^{2}, \ldots, X^{n}\right)$; each agent may use the whole or a limited set of the elements of $X$; this sub-set is noted $X_{A_{i}}$. Each element of this vector is a bounded value. Besides, the set of agents may use a fixed set of basic behaviors $B=\left\{B_{1}, B_{2}, \ldots, B_{p}\right\}$. At each time, the result of the decision taken by the master agent $A_{1}$ is the final execution of an element of $B$.

For each agent $A_{i}$, a prior knowledge is introducted before the learning process, which fixes the set of choices (call another agent or execute a basic task) $S_{A_{i}}=\left\{C_{i}^{1}, \ldots, C_{i}^{l}\right\}$ the agent can make to fulfill its constraints given at each time by a binary feedback signal $r_{A_{i}}$. Each decision $C_{i}^{k}$ of $S_{A_{i}}$ is associated to a branching priority $P_{i}^{k}$, which is a positive integer.

The learning process of $A_{i}$ consists of an on-line building of a mapping between the $X_{A_{i}}$ data and the elements of $S_{A_{i}}$. To do this, $A_{i}$ utilizes perceptual areas $Z_{i}^{k}$, each one linked to an element $C_{i}^{k}$ of $S_{A_{i}}$.

Each $Z_{i}^{k}$ is a continuous space generated par the whole set of possible $X_{C_{i}^{k}}$. It is divided into a set of boxes $B o x_{i, k}^{j, j \in\{1, \ldots, b\}}$ made up accordingly to the following set of equations:

$$
\left\{\begin{array}{l}
\forall k \in\{1, \ldots, l\} \bigcup_{j \in\{1, \ldots, b\}} B_{0 x}^{j}=Z_{i, k}^{k} \\
\forall k \in\{1, \ldots, l\} \bigcap_{j \in\{1, \ldots, b\}} B^{j} x_{i, k}^{j}=\emptyset \\
B o x_{i, k}^{j}=\left\{X_{C_{i}^{k}}=\left(X_{C_{i}^{k}}^{1}, \ldots, X_{C_{i}^{k}}^{k}\right) /\right. \\
\left.\forall j \in\left\{1, \ldots, n_{C_{i}^{k}}\right\}, m_{i, k}^{j} \leq X_{C_{i}^{k}}^{j}<M_{i, k}^{j}\right\}
\end{array}\right.
$$

Thus, each box $B o x_{i, k}^{j}$ is parameterized by $n_{C_{i}^{k}}$ couples of values $\left(m_{i, k}^{j}, M_{i, k}^{j}\right)$ which are the boundary values for each perceptive signal used when $C_{i}^{k}$ is called. 


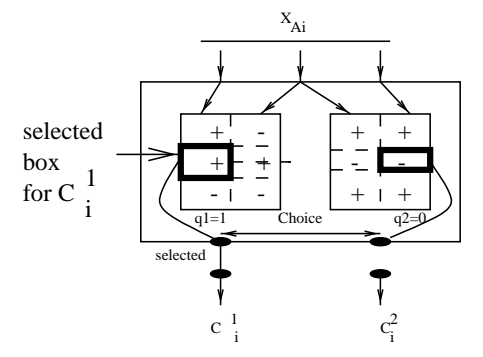

Figure 3: Decision-making of an agent $A_{i}$

Each box is associated with a binary quality $q_{i, k}^{j} \in$ $\{0,1\}$, which is the estimated quality of the decision made by the agent $A_{i}$ to call $C_{i}^{k}$.

Having received the set of the $l$ signals $X_{C_{i}^{k}}, A_{i}$ can know precisely which boxes $B o x_{i, k}^{j}$ are fired, therefore which quality $q_{i, k}^{j}$ is associated to each one of the possible choices. So, the decision linked to the best quality can be taken (fig. 3).

\subsection{Creating the perceptive graph}

The objective is to evaluate the impact of a choice among the $C_{i}^{k}$ on the evolution of the perception signal $X_{A_{i}}$ received by the agent $A_{i}$. To do so, while a failure signal is not sent, the agent is associated to a perceptive state $P_{A_{i}}\left(X_{A_{i}}(t)\right)=\left(j_{1}, \ldots, j_{l}\right)$ where each $j_{k}$ is an integer which is defined by the relationship $X_{C^{k}} \in B o x_{i, k}^{j_{k}}$. When a failure is detected by the way of $r_{A_{i}}$, the agent is in the special state $P_{e r r}$.

Thus, the agent $A_{i}$ has a finite number of states, including an ending state $P_{\text {err }}$. The dynamics of the system makes the agent move from one state to another, according to his choice made among the elements of $S_{A_{i}}$. This evolution is handled by the on-line building of a graph (fig. 4) which node $N_{P}$ are associated to a particular perceptive state already reached by the agent. The nodes are linked to a quality $q_{A_{i}}\left(X_{A_{i}}\right)$. A special node is associated with the state $P_{\text {err }}$.

If the agent is not in the state $P_{\text {err }}$, the effect of the decision of using $C_{i}^{k}$ is the transition from the state $P_{A_{i}}\left(X_{A_{i}}\right)$ to the transitory state $P_{C_{i}^{k}}\left(X_{C_{i}^{k}}\right)$ linked to the quality $q_{C_{i}^{k}}\left(X_{C_{i}^{k}}\right)=q_{i, k}^{j_{k}}$. Then, the reaction of the dynamics of the system when executing $C_{i}^{k}$ modifies the perception $X_{A_{i}}$, allowing the agent to move to another perceptive state at time t' (arc linking $P_{C_{i}^{k}}$ and $\left.P_{A_{i}}\left(X_{A_{i}}\left(t^{\prime}\right)\right)\right)$.

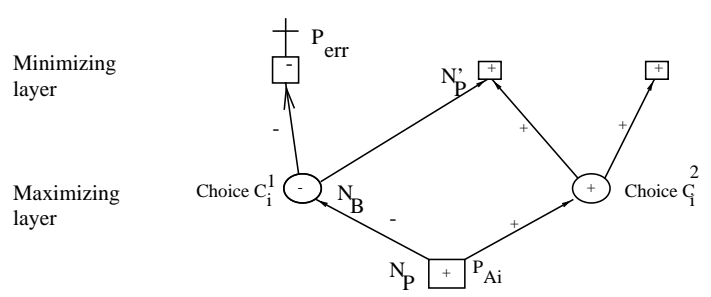

Figure 4: Detail of the graph associated to the learning process of the agent $A_{i}$. The current perceptive state is $P_{A_{i}}$. The past experience of the agent allows it to detect the transition to 3 different perceptive states from $P_{A_{i}}$ when the choice $C_{i}^{1}$ is made. The qualities are shown by some + and -, fitting the consistency law derived from the minimax algorithm.

\subsection{Consistency law.}

The problem which consists on taking a decision according to the fulfillment of constraints with a given dynamics of the system may be seen as a two players game: the agent $A_{i}$ and the dynamics. The aim of the agent is to never reach the losing state $P_{\text {err }}$, which quality $q_{P_{e r r}}$ is 0 . To do so, we use the minimax algorithm: the minimax searching tree is the perceptual graph and the evaluation function values are the qualities.

The consistency law applied for each node of the graph is given by the following two relationships. The first one is dealing about the perceptive nodes $N_{P}$, whereas the second one is applied to the transitory nodes $N_{B}$ associated to a decision-making when the perceptive state of the agent is $N_{P}$ :

$$
\begin{aligned}
& q_{N_{P}}=\max _{N_{i} \in \operatorname{Child}\left(N_{P}\right)}\left\{q_{N_{i}}\right\} \\
& q_{N_{B}}=\min _{N_{i} \in \operatorname{Child}\left(N_{B}\right)}\left\{q_{N_{i}}\right\}
\end{aligned}
$$

Where $\operatorname{Child}\left(N_{P}\right)$ is the set of the children of $N_{P}$ in the graph and $C h i l d\left(N_{B}\right)$ is the set of the children of $N_{B}$.

\subsection{Using the consistency law to learn.}

As soon as an arc from a node $N_{B}$ to a node $N_{P}^{\prime}$ is created while the dynamics makes the perceptual data of the agent evolve, $\operatorname{Child}\left(N_{B}\right)$ is modified, therefore the consistency relationship could be broken. In that case, the value of the quality of $N_{B}$ is forced in order to respect the equation (2). If $q_{N_{B}}$ is modified, the value of the quality associated with the father $N_{P}$ could be consequently modified due to the equation (1). A 
sequence of modifications may then happen, leading to a backpropagation of the prior modification. This ends as soon as the consistency law is fulfilled by the qualities of all the nodes.

\subsection{Global learning algorithm.}

Step 1- Retrieval of the node $N_{P}$ linked to the perceptive state of the agent: $P_{A_{i}}\left(X_{A_{i}}(t)\right)$. If it does not exist, create it and create the nodes $N_{B}$ associated to the choices $C_{k}^{i}$.

Step 2- Decision-making: choice of $C_{\max }$ among the elements of $S_{A_{i}}$, which associated quality is maximal. If two or more elements of $S_{A_{i}}$ have the same maximal quality, those which priority is maximal are chosen.

Step 3- Execution of $C_{\max }$ (transitory node $N_{B}$ ) and retrieval of the feedback signal $r_{A_{i}}$, while the perceptive state remains unchanged and no failure due to $A_{i}$ is detected. If another agent is utilized, the same algorithm is recursively called for it.

Step 4- Retrieval of the current perceptive node $N_{P}^{\prime}$. If the arc from $N_{B}$ to $N_{P}^{\prime}$ does not exist, create it and use the consistency law on this arc.

At each time, the first call to this algorithm is carried out for the master agent $A_{1}$.

\section{Validation experiments.}

\subsection{Context of the experiments.}

The experiments have been carried out with the help of the Khepera simulator. Khepera (fig. 5) is a small mobile robot developed at Ecole Polytechnique Fédérale de Lausanne (EPFL) which has a circular shape featuring $55 \mathrm{~mm}$ in diameter. It possesses 8 infrared sensors $s_{1}, \ldots, s_{8}$, allowing the measurement of distances in a short range from about $1 \mathrm{~cm}$ to $5 \mathrm{~cm}$ and the values they give ranges from 0 (no obstacle found) to 1024 (an obstacle is very near).

The Khepera simulator reproduces the imperfections of the sensors, so that it has been noticed that the experimental results deduced from the real and the simulated Khepera are very close.

In the following experiments, the simulated robot is controlled by receiving the values of the linear speed $l s_{1}$ and $l s_{2}$ of its two wheels. These values ranges from -10 to 10 , corresponding to a maximal speed of about $40 \mathrm{~mm} / \mathrm{s}$.

The objective is to build a goal-seeking behavior, making the hypothesis that the absolute coordinates

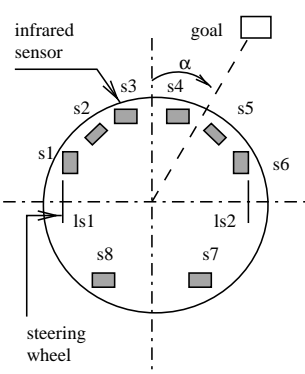

Figure 5: The miniature mobile robot Khepera.

\begin{tabular}{|c|c|c|c|}
\hline Behavior & meaning & $l s_{1}$ & $l s_{2}$ \\
\hline \hline$B_{1}$ & Move forward & 3 & 3 \\
\hline$B_{2}$ & Move to the right & 2 & 0 \\
\hline$B_{3}$ & Move to the left & 0 & 2 \\
\hline$B_{4}$ & Turn on the right & 2 & -2 \\
\hline$B_{5}$ & Turn on the left & -2 & 2 \\
\hline
\end{tabular}

Table 1: Basic behaviors utilized in the experiments. The $l s_{1}$ and $l s_{2}$ values come without any unit.

of both the goal and the robot are supposed to be precisely known at each time. The obstacle avoidance is performed by a wall following behavior, divided into two sub-tasks: follow the wall on the left and follow the wall on the right.

The agent possesses 4 input signals: $X=$ $\left(d_{\text {left }}, d_{\text {forward }}, d_{\text {right }}, \alpha\right) . \quad \alpha$ is the angle between the robot direction and the goal. The value of the angle is supposed to be known at each time. $d_{\text {left }}=\max \left(s_{1}, s_{2}\right), d_{\text {forward }}=\max \left(s_{3}, s_{4}\right), d_{\text {right }}=$ $\max \left(s_{5}, s_{6}\right)$

Five basic behaviors have been chosen, which are linked to a couple $\left(l s_{1}, l s_{2}\right): B=\left\{B_{1}, B_{2}, B_{3}, B_{4}, B_{5}\right\}$. Their specification is given by table 1 .

The robot possesses three internal binary feedback signals: BUMP, FWL and FWR. BUMP is equal to 1 if the robot has bumped into an obstacle, else it is equal to 0 . FWL (resp. FWR) is equal to 0 if the $d_{l e f t}$ (resp. $d_{\text {right }}$ ) value has remained smaller 10 for more than 30 learning steps.

\subsection{High level goal-seeking algorithm.}

The goal-seeking strategy followed by the robot is a high-level algorithm in which three contexts are considered: "reach the goal", "follow the wall on the left" and "follow the wall on the right".

[reach the goal]

If the goal is behind the robot and it can go forward, $B_{1}$ is executed. 
If the robot is near from an obstacle and the goal is on the same direction, it switches to a context [follow the wall]

[follow the wall on the right (resp.left)]

If (the goal is on the left (resp. right) side of the robot and it can go on the left (resp. right) without colliding) or (the goal is behind the robot and it can go forward without colliding), it switches its context to [reach the goal].

Else the agent associated to a "follow the wall on the right (resp. left)" behavior is utilized.

\subsection{Specification of the learning agents.}

The algorithm described in the last paragraph shows that the strategy of the robot uses its perceptive data. The learning process focuses on their management. Both the hierarchical set of the learning agents and their internal constraints, using BUMP, FWR and FWL, are given by fig. 6 . There are two master agents $A_{1}$ and $A_{1}^{\prime}$, which respectively accomplish the "follow the wall on the right" and "follow the wall on the left" tasks. $A_{2}$ and $A_{2}^{\prime}$ agents must avoid obstacles respectively by moving on the left and by moving on the right. The perceptive areas associated to these four agents are three dimensional continuous spaces generated by $\left(d_{\text {left }}, d_{\text {forward }}, d_{\text {right }}\right)$; they are regularly divided into $4 \times 4 \times 4=64$ boxes.

The quality of the box fired in the perceptive area of the agent $A_{2}$ associated to the choice of $B_{3}$ is used by the robot to know if it can turn on the left without colliding (see paragraph 3.2). In the same way, the quality of the box fired in the perceptive area of the agent $A_{2}^{\prime}$ associated to the choice of $B_{2}$ is used by the robot to know if it can turn on the right without colliding.

\subsection{Learning protocol.}

A learning process, which consists of trials/failures steps, is developed in the environment given by fig. 7 . A trial ends when a failure is detected by one of the agents or when the goal is reached. As a first stage of the global learning, the low level agents $A_{2}$ and $A_{2}^{\prime}$ are utilized independently without taking the high level algorithm into account. To do this, the position of the robot is randomly initialized in the free space of the environment at the beginning of the trial, which ends when BUMP turns to 1 or when 500000 consecutive learning steps have been accomplished without any failure. As a second stage of the learning process, $A_{1}$ and $A_{1}^{\prime}$ are utilized, using $A_{2}$ and $A_{2}^{\prime}$ obtained at the end of the first stage. The position of the robot is initialized near a wall. At the end of the second stage,

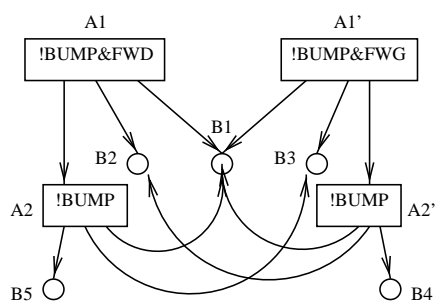

Figure 6: Hierarchical set of agents used by Khepera.

the 4 agents are utilized in the context of the global goal-seeking algorithm described in paragraph 3.2 .

\subsection{Results.}

10 learning attempts have been done for $A_{2}$. All the attempts were successful, ending after 35 up to 42 trials. Fig. 8 shows the evolution of the number of consecutive learning steps without failure. It is noticed that the duration of a trial is a function of the number of nodes (fig. 9). It simply means that as soon as the agent has a wide perceptive experience, it is able to respect its constraints with making very few mistakes. According to the perceptive areas division process (see paragraph 3.3), the maximal number of nodes for all the agents is $64+3 \times 64+1=257$. This number is nearly reached at the end of the learning stage. As a consequence, we have noticed that the failures that come within the learning process of master agent $A_{1}$ using $A_{2}$ are not the result of a mistake made by $A_{2}$ : they all are due to misleading decisions taken by $A_{1}$. The learning period of $A_{1}$ has successfully ended after 25 up to 33 trials. When all the agents have successed in their own learning stage, they are used by the global goal-reaching algorithm. The final result is given by fig. 7. Here, it must be underlined that the wall-following behavior task accomplished by $A_{1}$ and $A_{1}^{\prime}$ was not immediately perfect. Indeed, they have been facing new perceptive situations due to the switchings between the three different contexts. After the trial 10, no failure has been detected up to the finishing trial 20 .

\section{Discussion and future work.}

The learning method and the algorithm allow a hierarchical set of agents to incrementally learn. According to its perception, the aim of each of the agents is respect its internal binary constraints. To do so, it must decide to execute a basic task or to call another 


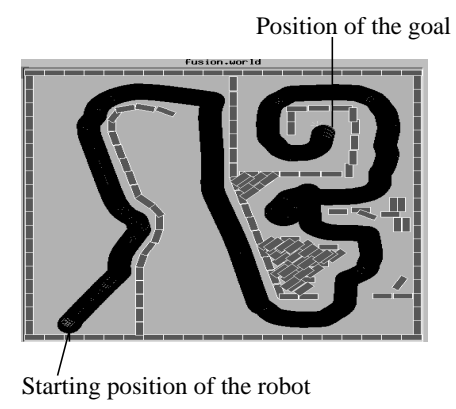

Figure 7: Goal-reaching behavior in the Khepera simulator environment.

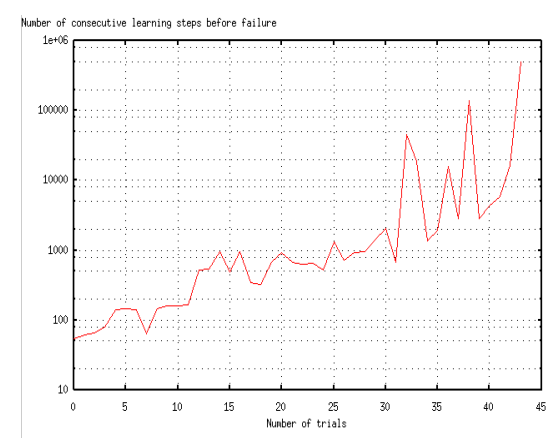

Figure 8: Evolution of the number of consecutive learning steps without a failure of the agent $A_{2}$.

agent, which is lower in the hierarchy, to let it choose by itself.

Using a simulated Khepera robot which aim is to learn a safe goal-reaching behavior, it has been shown that the hierarchical set of agents created for this task are able to learn at any time they are utilized. Thus, although they principally learn during their own learning stage, they can evolve if they are used in a high level algorithm framework where they have to face new perceptive situations. Besides, the algorithm can cope with very noisy perceptive data produced by the infrared sensors of Khepera.

The quality associated to each node of these graphs can be used to statically recognize some perceptive situations. Moreover, it should be possible to use the perceptive graph in a dynamic environment recognizing process: the execution of the wall-following task generates a particular cyclic sequence of perceptive states for the wall-following agent. So, we are thinking of adding new kind of nodes associated to cycles into the graph to cope with dynamic recognition of situations.

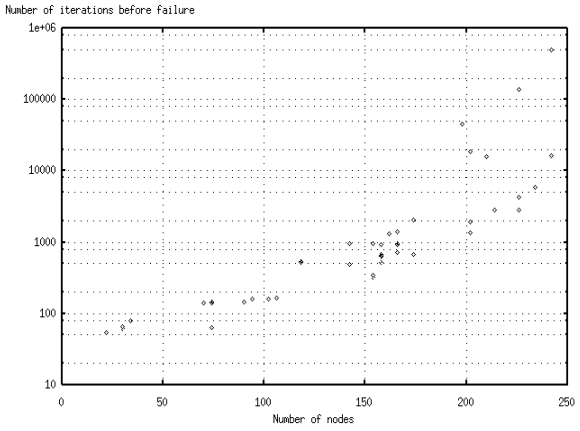

Figure 9: Relationship between the number of nodes within the perceptive graph of $A_{2}$ and the number of consecutive learning steps without a failure.

\section{References}

[1] C.J.C.H. Watkins. "Learning from delayed rewards". PhD thesis, King's College, University of Cambridge, May 1989.

[2] L-J. Lin. "Self-Improving Reactive Agents Based on Reinforcement Learning, Planning and Teaching". In Machine Learning, (8), pp.293-321, 1992.

[3] M.Asada, S.Noda and K.Hosoda. "Action-Based Sensor Space Categorization for Robot Learning". In IROS 96, pp1502-1509, 1996.

[4] H.Bersini and V.Gorrini. "Three Connectionist Implementations of Dynamic Programming for Optimal Control: A Preliminary Comparative Analysis". In Workshop on NN for Id. Ctrl. Rob. , 1996.

[5] M.Asada, E.Uchibe, S.Noda, S.Tawaratsumida and K.Hosoda. "Coordination of Multiple Behaviors Acquired bya Vision-Based Reinforcement Learning". In IROS 94, pp917-924, 1994.

[6] E.Rich, "Artificial Intelligence". McGraw-Hill, 1983.

[7] F.Mondada, E.Franzi and P.Lenne "Mobile robot miniaturisation: a tool for investigation in control algorithms". In Proc.3. Int. Symposium on Experimental Robotics, Kyoto, 1993.

[8] O.Michel. Khepera Simulator Package version 2.0: Freeware mobile robot simulator written at the University of Nice Sophia-Antipolis. Downloadable at http://wwwi3s.unice.fr/ ${ }^{\sim}$ om/khep-sim.html , 1996. 